\title{
AVALIAÇÃo DE CONTROLES PID ADAPTATIVOS PARA UM SISTEMA DE AQUECIMENTO RESISTIVO DE ÁGUA ${ }^{1}$
}

\author{
Maria Isabel BERTO ${ }^{2 *}$, Fabiana Rodrigues de SÁ ${ }^{2}$ Vivaldo SILVEIRA JR. ${ }^{2}$
}

\begin{abstract}
RESUMO
O trabalho consiste na implementação de um controle convencional PID/SISO-feedback para obter um ajuste fino na temperatura de entrada da água de aquecimento em um processo de pasteurização. Para isto utilizou-se uma resistência de 2500 Watts instalada na linha do fluido secundário da seção de aquecimento do pasteurizador e um Pt100 para a medição de sua temperatura. Como o comportamento desta temperatura em função de uma mesma perturbação degrau de potência na resistência é dependente da vazão de trabalho, objetivou-se encontrar um controle único para que a mesma fosse mantida no set-point desejado na faixa de operação de vazão da água do processo $(300$ a $700 \mathrm{~L} / \mathrm{h})$. Três sintonias para o controlador adaptativo PID foram testadas: a primeira consistiu na implementação de uma função adaptativa dos parâmetros PID, ajustada através dos valores individuais obtidos para cada vazão de trabalho conforme metodologia da curva de reação do processo; a segunda consistiu em configurar os parâmetros do PID com os valores médios destes calculados individualmente para cada vazão, e a terceira consistiu na sintonia através de uma função adaptativa ajustada pelos parâmetros de sintonia obtidos pela metodologia de Aström \& Hägglund. A avaliação do desempenho das sintonias dos controladores adaptativos foi realizada por comparação dos valores dos índices de erro, obtidos por perturbações do sistema em malha fechada na vazão de água. Os resultados obtidos mostraram que dentre as sintonias testadas, a terceira sintonia, popularmente conhecida como "Bang-Bang", apresentou menores oscilações e os menores valores dos índices de erros.

Palavras-chave: controle adaptativo; aquecimento resistivo; PID; pasteurização; sintonia.
\end{abstract}

\section{SUMMARY}

EVALUATION OF ADAPTIVE PID CONTROLS FOR A RESISTIVE SYSTEM OF HEATING WATER. The aim of this work is to implement a conventional PID/SISO feedback control to obtain a fine adjustment of the water inlet temperature at a pasteurization process. For that, a resistance of 2500 Watts and a Pt100 to measure the temperature were installed in the water inlet line of the pasteurizer heating section. As the water temperature behavior according to the same step change on the potency of the resistance depends on the working flow rate, a single controller was designed to keep this temperature at its desirable set-point, for the water flow rate, within the range of 300 to $700 \mathrm{~L} / \mathrm{h}$. Three different tunings for the PID were tested: the first consisted on the implementation of a function for the calculation of the PID parameters fitted to individual values obtained from each flow rate, according to process reaction curve methodology; the second consisted on using the PID parameters calculated as the average of these individual values; at the third tuning, an adaptive function fitted with the individual parameters obtained with Aström \& Hägglund methodology was used. The performance evaluation of the configured PID controllers was carried out by comparing the error index values, obtained after disturbances in the water flow rate in the closed loop system. The error indexes calculated after step changes in the water flow rate were used to evaluate the tunings. The results have shown that the third tuning, called "Bang Bang" presented minor oscillations and smaller error indexes compared to the other two methods.

Keywords: adaptive control; resistive heating; PID; pasteurization; tuning.

\section{1 - INTRODUÇÃO}

A pasteurização de fluidos é amplamente utilizada em indústrias alimentícias com o objetivo de proporcionar a desativação enzimática, evitar o crescimento microbiológico e estender a vida-de-prateleira dos alimentos. O processo consiste em aquecer o fluido alimentício até a temperatura de pasteurização e mantêlo nesta temperatura por um período suficiente para que as reações indesejáveis sejam inativadas e, em seguida, resfriá-lo até sua temperatura de estocagem. O binômio tempo/temperatura de pasteurização depende das características físico-químicas de cada produto. No caso do suco de laranja natural, estudos como os

\footnotetext{
1. Recebido para publicação em 10/11/2003. Aceito para publicação em 28/05/2004 (001243).

2. Laboratório de Automação e Controle de Processos de Alimentos, Depto. Engenharia de Alimentos, Universidade Estadual de Campinas-UNICAMP, Monteiro Lobato, 80, Caixa Postal 6121, CEP 13081-970, Barão Geraldo, Campinas, São Paulo, Brasil. Tel: +55 (19) 3788-4086. Fax: +55 (19) 3788-4027.E-mail: isabel@berto.com.br, vivaldo@fea.unicamp.br * A quem a correspondência deve ser enviada.
}

de KIMBALL [7] e CORREA NETO [3] mostraram que a pasteurização a temperaturas relativamente baixas $\left(65,6^{\circ} \mathrm{C}\right.$ ou $\left.72^{\circ} \mathrm{C}\right)$ são suficientes para a inativação microbiana, porém são ineficazes para manter a estabilidade do suco em relação a sua turbidez característica, considerada um importante parâmetro de aceitação pelo consumidor. Por este motivo, binômios com temperaturas superiores a $90^{\circ} \mathrm{C}$ são usualmente empregados nas indústrias processadoras deste suco. Independentemente do produto a ser pasteurizado e do binômio utilizado, o imprescindivel neste processo é atingir a temperatura de pasteurização e mantê-la durante sua passagem pelo tubo de retenção. Diante deste fato, muitos trabalhos são enfocados para o controle desta temperatura através de diferentes estratégias de controle, como NEGIZ et al. [8], SCHLESSER et al. [10], IBARROLA et al. [6]. Na maioria destes trabalhos, vapor superaquecido foi utilizado como meio aquecedor e o controle da temperatura de pasteurização do produto é realizado pelo controle da vazão deste vapor.

No trabalho de BERTO [2] o processo de pasteurização montado consistiu em um trocador de calor a placas de 3 seções (regeneração, aquecimento e resfria- 
mento) e em um tubo de retenção para a pasteurizar o produto numa vazão $150 \mathrm{~L} / \mathrm{h}$. O binômio tempo/temperatura utilizado foi de $91^{\circ} \mathrm{C} / 40 \mathrm{~s}$, baseado nos trabalhos de por EARGMAN \& ROUSE [5], KIMBALL [7] e CORREA NETO [3]. Água a alta temperatura $\left(96,6^{\circ} \mathrm{C}\right)$ a $483 \mathrm{~L} / \mathrm{h}$ foi responsável por aquecer o produto, enquanto que solução de propileno-glicol a $25 \% \mathrm{~m} / \mathrm{m},-1,7^{\circ} \mathrm{C}$ e $1920 \mathrm{~L} / \mathrm{h}$ o resfriou em torno de $9^{\circ} \mathrm{C}$. Como o controle da temperatura de pasteurização do produto foi realizado pela manipulação da vazão da água de aquecimento, a manutenção da temperatura deste fluido secundário no seu set-point $\left(96,6^{\circ} \mathrm{C}\right)$ foi fundamental para que o processo ocorresse com sucesso.

Para a manutenção da temperatura da água de aquecimento, sob diversas vazões de trabalho, foram implementados dois controladores no processo. O primeiro foi implementado para manter o tanque de armazenamento da água de aquecimento na faixa de $\pm 2^{\circ} \mathrm{C}$ do set-point desejado através da implementação de um controlador tipo liga-desliga na resistência de 2500 Watts instalada no mesmo. Para o ajuste fino da temperatura, outra resistência de 2500 Watts, comandada pela unidade de potência, foi instalada na linha de água a $20 \mathrm{~cm}$ da entrada da seção de aquecimento do pasteurizador.

Neste contexto, o objetivo deste trabalho foi sintonizar um controle do tipo PID/SISO-feedback para este ajuste fino, onde potência da resistência da linha da água de aquecimento foi a variável manipulada e a sua temperatura, a variável controlada. Como o comportamento desta temperatura em função de uma mesma perturbação degrau na resistência é dependente da vazão de trabalho, objetivou-se encontrar um controle único para que a mesma fosse mantida no set-point desejado para a faixa de operação de vazão da água do processo (300 a 700L/h).

Apesar das sintonias propostas na literatura e aplicadas neste trabalho serem classificadas como sugestões iniciais para os parâmetros dos controladores, objetivou-se comparar o desempenho das mesmas, com aplicação direta dos resultados, sem nenhum ajuste posterior nos valores dos parâmetros calculados por cada método.

\section{1 - Métodos de sintonia}

Dentre as metodologias de sintonia dos controladores convencionais PID, as mais utilizadas são: (a) A metodologia curva de reação do processo proposta por Cohen \& Coon; (b) A Metodologia de sintonização da malha, proposta por Ziegler-Nichols, baseada na obtenção de uma oscilação sustentada na variável de processo e (c) Metodologia de ASTRÖM \& HÄGGLUND [1] que é uma modificação da metodologia (b).

Segundo COUGHANOWR \& KOPPEL [4] a primeira metodologia consiste, com o sistema em malha aberta, aplicar uma perturbação degrau na variável manipulada e obter a curva reação do processo em formato sigmoidal.
Através dos parâmetros gráficos obtidos, mostrados na Figura 1, calculam-se os parâmetros dos controladores conforme as relações propostas em literatura por Cohen \& Coon ou por Ziegler-Nichols (Tabela 1), onde "K" é a relação entre o ganho estático $(\mathrm{Bu})$ e a amplitude da perturbação (A).

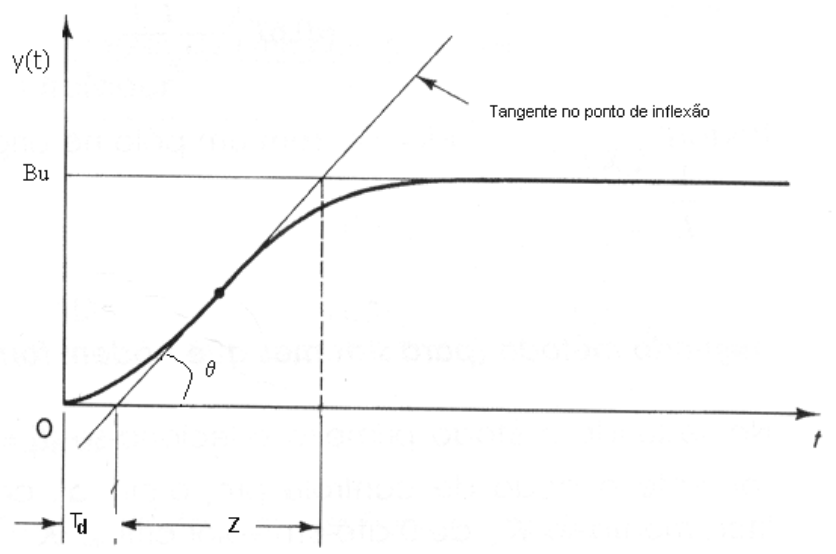

FIGURA 1. Curva típica de reação de processo

TABELA 1. Parâmetros do PID conforme regra de ajuste de Ziegler-Nichols

\begin{tabular}{cccc}
\hline Controlador & $\mathrm{K}_{\mathrm{c}}$ & $\tau_{\mathrm{i}}$ & $\tau_{\mathrm{D}}$ \\
\hline $\mathrm{P}$ & $\frac{1}{\mathrm{~K}} \frac{\mathrm{Z}}{\mathrm{T}_{\mathrm{d}}}$ & $\infty$ & 0 \\
$\mathrm{PI}$ & $\frac{1}{\mathrm{~K}} 0,9 \frac{\mathrm{Z}}{\mathrm{T}_{\mathrm{d}}}$ & $\frac{\mathrm{T}_{\mathrm{d}}}{0,3}$ & 0 \\
PID & $\frac{1}{\mathrm{~K}} 1,2 \frac{\mathrm{Z}}{\mathrm{T}_{\mathrm{d}}}$ & $2 \mathrm{~T}_{\mathrm{d}}$ & $0,5 \mathrm{~T}_{\mathrm{d}}$ \\
\hline
\end{tabular}

A segunda metodologia, de sintonização da malha, foi proposta por Ziegler-Nichols, sendo aplicada para sistemas que podem tornar-se instáveis [4]. Este método consiste em, com o sistema em malha fechada, perturbar o set-point $\mathrm{e}$ incrementar o valor de $\mathrm{K}_{c}$ (constante proporcional do PID), considerando apenas o controlador proporcional, ou seja, com valores de $\tau_{\mathrm{i}}=\infty \mathrm{e}$ $\tau_{d}=0$, até que o sinal de saída exiba uma oscilação sustentada. Este valor de $\mathrm{K}_{\mathrm{c}}$ é chamado de valor de ganho crítico $\left(\mathrm{K}_{\mathrm{cr}}\right)$ e o período de oscilação correspondente, determinado graficamente, é chamado de período crítico $\left(\mathrm{P}_{\mathrm{cr}}\right)$. Como o aumento do ganho do controle proporcional $\left(\mathrm{K}_{\mathrm{c}}\right)$ deve ser efetuado gradativamente, na prática isto proporciona uma relativa demora na determinação do ganho crítico. Assim sendo, ASTRÖM \& HÄGGLUND [1] propuseram uma metodologia de sintonia que consiste em obter esta oscilação sustentada em torno de um valor de referência. Para isto, com o sistema em malha aberta, a variável manipulada é chaveada em dois valores convenientemente escolhidos, numa freqüência constante. A Figura 2 ilustra o comportamento da variável manipulada e a reação do processo a este chaveamento. 
Neste caso, o ganho crítico pode ser calculado como mostra a equação (1), onde $2 \mathrm{~h}$ é a amplitude de chaveamento e 2I a amplitude, pico a pico, da oscilação observada. Este método também é conhecido como "bang-bang”.

$\mathrm{K}_{\mathrm{cr}}=\frac{4 \mathrm{~h}}{\pi \mathrm{I}}$

Neste caso, as constantes do PID são também calculadas conforme proposto por Ziegler-Nichols na metodologia de sintonização da malha, para sistemas que podem se tornar instáveis, conforme mostra a Tabela 2.

TABELA 2. Constantes recomendadas por Ziegler-Nichols de sintonização da malha

\begin{tabular}{lccc}
\hline & \multicolumn{3}{c}{ Constantes } \\
\hline Modos de controle & $\mathrm{K}_{\mathrm{c}}$ & $\tau_{\mathrm{i}}$ & $\tau_{\mathrm{d}}$ \\
Proporcional $(\mathrm{P})$ & $0,5 \mathrm{~K}_{\mathrm{cr}}$ & $\infty$ & 0 \\
Proporcional-integral (PI) & $0,4 \mathrm{~K}_{\mathrm{cr}}$ & $0,8 \mathrm{P}_{\mathrm{cr}}$ & 0 \\
Proporcional-integral-derivativo (PID) & $0,6 \mathrm{~K}_{\mathrm{cr}}$ & $0,5 \mathrm{P}_{\mathrm{cr}}$ & $0,125 \mathrm{P}_{\mathrm{cr}}$ \\
\hline
\end{tabular}

\section{2 - MATERIAIS E MÉTODOS}

Os testes experimentais foram realizados na planta-piloto de pasteurização de suco de laranja natural montada por BERTO [2], instalada no Laboratório de Automação e Controle de Processos de Alimentos (LACPA) da Faculdade de Engenharia de Alimentos da UNICAMP, Campinas - SP. A temperatura da água de aquecimento foi controlada através do acionamento de uma resistência monofásica de 2500 Watts instalada na linha a $20 \mathrm{~cm}$ da entrada do trocador, por uma unidade de potência a SCR, modelo MUP-100 (Markare Equipamentos Ltda). A vazão da água de aquecimento foi imposta por um variador de freqüência VLT ${ }^{\circledast}$ série 2800 (Danfoss S/A) instalado no motor da bomba de água. $\mathrm{O}$ comando da rotação da bomba e da unidade de potência foi realizado via comunicação Fieldbus, pelo supervisório AIMAX for Windows ${ }^{\circledR}$, através de um conversor fieldbuscorrente, modelo FI 302 (Smar Equipamentos Ltda). A medição de temperatura foi realizada através de um Pt100 (Markare Equipamentos Ltda) instalado na linha da água de aquecimento, após a resistência monofásica.

\section{1 - Sintonia dos controladores}

Primeiramente foram obtidas as curvas de reação do processo após perturbações degrau de $100 \%$ na potência da resistência monofásica com as seguintes condições iniciais: temperatura da água ambiente $\left(25^{\circ} \mathrm{C}\right)$ com a resistência da linha desligada para seis vazões de trabalho: 300, 330, 400, 500, 600 e 700L/h. A partir da estabilização das condições iniciais, em cada vazão foi realizada a perturbação degrau de $100 \%$ na resistência da linha. Para não haver interferência do retorno da água na temperatura inicial, seu retorno foi desviado do tanque de armazenamento. Com as curvas de reação obtidas para cada vazão foi realizada a caracterização do comportamento transiente do processo res- pectivo através do cálculo dos parâmetros gráficos Bu, $\mathrm{T}_{\mathrm{d}}$ e $Z$, mostrados na Figura 1.

Como proposta de implementação do controlador adaptativo, foram testadas três sintonias para o controlador PID, sendo que as duas primeiras utilizam as constantes calculadas pelos parâmetros gráficos das curvas de reação do processo e a terceira é baseada no método de sintonia de ASTRÖM \& HÄGGLUND [1].

\subsection{1 - Sintonia adaptativa, pelo método da Curva de Reação do Processo}

Neste caso foi feita a implementação de uma função adaptativa para o cálculo das constantes do PID $\left(\mathrm{K}_{\mathrm{c}}, \tau_{\mathrm{i}} \mathrm{e} \tau_{\mathrm{d}}\right)$, ajustada através dos valores individuais obtidos para cada vazão de trabalho. Estas constantes individuais foram calculadas conforme o método da curva de reação do processo, pelos dos parâmetros gráfi$\cos \mathrm{Bu}, \mathrm{T}_{\mathrm{d}}$ e $\mathrm{Z}$ (Figura 1) obtidos em cada uma das seis curvas de reação, utilizando as relações apresentadas na Tabela 1. A função adaptativa para ajuste das constantes em função da vazão de trabalho foi uma equação de segundo grau, por ter apresentado maior coeficiente de correlação.

\subsection{2 - Sintonia pelas constantes médias do PID}

Neste caso, foram calculadas as médias dos valores dos parâmetros encontrados pelo método da curva de reação de processo (item 2.1.1) para serem implementados diretamente no controlador PID.

\subsection{3 - Sintonia pelo método de ASTRÖM \& HÄGGLUND [1]}

Neste caso foi feita a implementação de uma função adaptativa ajustada pelos parâmetros de sintonia obtidos pela metodologia de ASTRÖM \& HÄGGLUND [1], para cada vazão de trabalho.



FIGURA 2. Oscilação sustentada obtida pela metodologia de ASTRÖM \& HÄGGLUND [1]

As condições iniciais destes ensaios para cada vazão de trabalho $(300,400,500,600,700 \mathrm{~L} / \mathrm{h})$ foram: temperatura da água a $25^{\circ} \mathrm{C}$ e potência da resistência desligada. A cada 30 segundos uma perturbação degrau de 
$\pm 50 \%( \pm 1250$ Watts $)$ na potência da resistência da linha foi imposta para a obtenção de uma oscilação sustentada na variável resposta (temperatura do fluido térmico) fosse obtida para cada vazão. A definição do período de imposição, variação da potência e da amplitude das mesmas, de 30 e $\pm 50 \%$, foi baseada no trabalho de SILVA [11] e em testes preliminares realizados, com o objetivo de obter uma oscilação sustentada mensurável na temperatura a ser controlada.

Através da resposta oscilatória foi obtido o valor da intensidade da resposta, "I" (Figura 2) e calculados os parâmetros dos controladores conforme a Tabela 2. Como se obteve um valor do ganho proporcional $\left(\mathrm{K}_{\mathrm{c}}\right)$ para cada vazão de trabalho, uma equação foi ajustada para seu cálculo em função da vazão para a implementação do terceiro controlador adaptativo proposto.

\section{2 - Avaliação do desempenho dos controladores}

A avaliação do desempenho dos três controladores implementados foi realizada através de perturbações na carga do sistema em malha fechada pelas vazões do fluido, conforme esquematiza a Tabela 3. As condições iniciais para esta avaliação foram os valores médios das faixas de vazão e de potência da resistência, ou seja, 500L/h e 50\%.

TABELA 3. Esquema de perturbações para avaliação dos controladores

\begin{tabular}{lc}
\hline \multicolumn{1}{c}{ Etapa } & Intensidade das variáveis \\
\hline Condição inicial & $23,0^{\circ} \mathrm{C}, 500 \mathrm{~L} / \mathrm{h}, 1250 \mathrm{~W}(50 \%)$ de resistência \\
Perturbação positiva na vazão (+) & $+50 \mathrm{~L} / \mathrm{h}$ \\
Perturbação positiva na vazão (++) & $+100 \mathrm{~L} / \mathrm{h}$ \\
Perturbação negativa na vazão (-) & $-50 \mathrm{~L} / \mathrm{h}$ \\
Perturbação negativa na vazão (--) & $-100 \mathrm{~L} / \mathrm{h}$ \\
\hline
\end{tabular}

O desempenho do controlador foi testado por 2,7 minutos em cada ensaio $\left(\tau_{\infty}\right)$. Foram comparados os valores de erro da estabilização da temperatura $\left(\mathrm{e}_{\infty}\right)$, o tempo e erro de sobre-sinal $\left(\mathrm{t}_{\mathrm{s}}, \mathrm{e}_{\mathrm{p}}\right)$, referentes aos valores obtidos no overshoot e os valores dos erros especificados a seguir:

Valor absoluto do erro (IAE): $\mathrm{IAE}=\int_{0}^{\infty}|e(t)| d t$

Valor absoluto do erro ao quadrado (ISE): ISE $=\int_{0}^{\infty}[e(t)]^{2} d t$

Valor do erro absoluto ponderado (ITAE): ITAE $=\int_{0}^{\infty} t \cdot|e(t)| d t$

\section{3 - RESULTADOS E DISCUSSÃO}

\section{1 - Análise da resposta transiente do desvio da temperatura sob perturbações na resistência da linha - Curvas de reação do processo}

A Figura 3 mostra a variação do desvio da temperatura (T- $\mathrm{T}_{0}$ ) constatada após a mesma perturbação de- grau de $100 \%$ (2500W) na potência da resistência da linha de água de aquecimento para as seis vazões de trabalho (300, 330, 400, 500, 600 e 700L/h) em função do tempo. Tanto a resposta transiente do desvio da variável de processo como o valor final de estabilização da temperatura, no segundo regime permanente para cada vazão, foram distintos. Assim, os valores dos parâmetros dos controladores PID $\left(K_{c}, \tau_{i}\right.$ e $\left.\tau_{d}\right)$ foram função da vazão de trabalho.

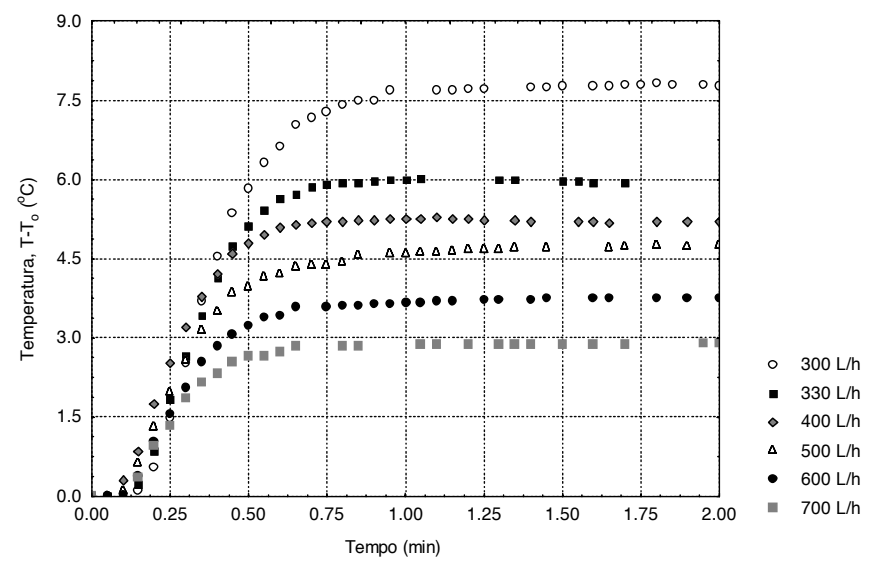

FIGURA 3. Curvas de reação do processo após perturbação de $100 \%$ na resistência para diferentes vazões de trabalho

A Tabela 4 mostra os valores dos parâmetros gráfi$\cos \mathrm{T}_{\mathrm{d}}$, Bu e $\mathrm{Z}$ encontrados pela reta tangente traçada à curva de reação do processo.

TABELA 4. Parâmetros gráficos das curvas de reação

\begin{tabular}{lcccccc}
\hline \multirow{2}{*}{ Parâmetro } & \multicolumn{6}{c}{ Vazão de trabalho (L/h) } \\
\cline { 2 - 7 } & 300 & 330 & 400 & 500 & 600 & 700 \\
\hline $\mathrm{T}_{\mathrm{d}}(\mathrm{s})$ & 10,31 & 9,19 & 5,41 & 5,32 & 6,35 & 6,46 \\
$\mathrm{Bu}\left({ }^{\circ} \mathrm{C}\right)$ & 7,78 & 5,94 & 5,17 & 4,72 & 3,76 & 2,91 \\
$\mathrm{Z}(\mathrm{s})$ & 24,61 & 20,88 & 20,63 & 24,12 & 21,97 & 18,70 \\
\hline
\end{tabular}

\section{2 - Sintonia dos controladores PID propostos}

\subsection{1 - Sintonia do controlador PID adaptativo, conforme método da curva de reação do pro- cesso}

Com as curvas de reação apresentadas na Figura 3, e os parâmetros de sintonia dos controladores PID da Tabela 4 foram ajustadas as equações de segundo grau em função da vazão do fluido para cada parâmetro do controlador PID adaptativo.

A Tabela 5 mostra os valores de $\mathrm{K}_{\mathrm{c}} \tau_{\mathrm{i}}$ e $\tau_{\mathrm{d}}$, que são os parâmetros proporcional, tempo integral e tempo derivativo, respectivamente, do controlador PID. A relação $\mathrm{T}_{\mathrm{d}} / Z$ é uma referência da adequação do controle PID configurado ao processo. Segundo OGUNNAIKE \& RAY [9], para um controlador PID estar adequado esta relação deve ser $0,1<\mathrm{T}_{\mathrm{d}} / Z<1$. 
TABELA 5. Constantes do PID para o controle da temperatura de água de aquecimento.

\begin{tabular}{lccccccc}
\hline \multirow{2}{*}{ Parâmetro } & \multicolumn{7}{c}{ Vazão de trabalho $(\mathrm{L} / \mathrm{h})$} \\
\cline { 2 - 8 } & 300 & 330 & 400 & 500 & 600 & 700 & Média \\
\hline $\mathrm{T}_{d} / \mathrm{Z}$ & 0,42 & 0,44 & 0,26 & 0,22 & 0,29 & 0,35 & \\
$\mathrm{~K}_{\mathrm{c}}\left({ }^{\circ} \mathrm{C} / \%\right)$ & 36,83 & 45,89 & 88,59 & $11,52.10$ & $11,04.10$ & $11,94.10$ & 86,05 \\
$\tau_{i}(\mathrm{~s})$ & 20,62 & 18,39 & 10,82 & 10,64 & 12,70 & 12,92 & 14,35 \\
$\tau_{d}(\mathrm{~s})$ & 5,16 & 4,60 & 2,71 & 2,66 & 3,18 & 3,23 & 3,59 \\
\hline
\end{tabular}

As funções ajustadas para os parâmetros dos controladores mostradas na Figura 4 são apresentadas nas equações (2) a (4).

$$
\begin{array}{ll}
\mathrm{K}_{\mathrm{c}}=-9,00 \cdot 10^{-4} \mathrm{~V}_{\mathrm{aq}}{ }^{2}+1,08 \mathrm{~V}_{\mathrm{aq}}-2,07.10^{2} & \mathrm{R}^{2}=0,9636 \\
\tau_{\mathrm{i}}=1,64 \cdot 10^{-4} \mathrm{~V}_{\mathrm{aq}}{ }^{2}+1,79.10^{-1} \mathrm{~V}_{\mathrm{aq}}+5,88 \cdot 10 & \mathrm{R}^{2}=0,8372 \\
\tau_{\mathrm{d}}=4,10.10^{-5} \mathrm{~V}_{\mathrm{aq}}^{2}+-4,47.10^{-2} \mathrm{~V}_{\mathrm{aq}}+1,47.10 & \mathrm{R}^{2}=0,8373
\end{array}
$$

As funções ajustadas para os parâmetros dos controladores PID são mostradas nas Figuras 4 e 5 :

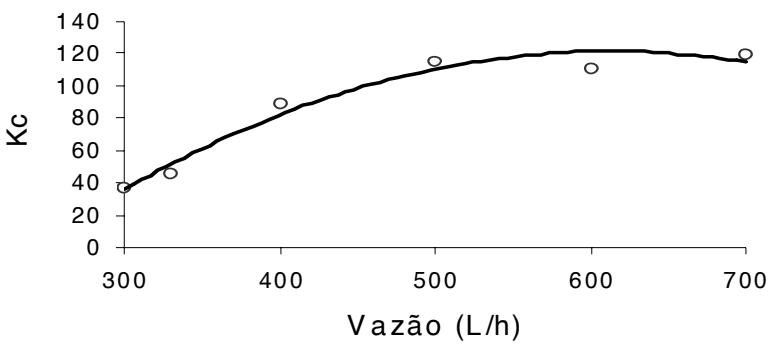

FIGURA 4. Ajuste dos valores de $\mathrm{K}_{\mathrm{c}}$ em função da vazão do fluido (Método da curva de reação)

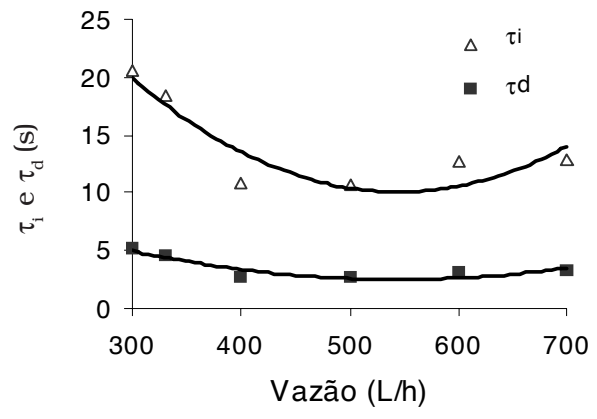

FIGURA 5. Ajuste dos valores de $\tau_{\mathrm{i}} \mathrm{e} \tau_{\mathrm{d}}$ em função da vazão (Método da curva de reação)

\subsection{2 - Sintonia do controlador com os parâ- metros médios}

Neste caso, os parâmetros do controlador PID implementados foram calculados pela média dos valores encontrados para cada vazão, conforme método da curva de reação do processo (Tabela 5). Assim, os valores dos parâmetros $\mathrm{K}_{\mathrm{c}} \tau_{\mathrm{i}}$ e $\tau_{\mathrm{d}}$ foram respectivamente $86,05^{\circ} \mathrm{C} / \%$; $14,35 \mathrm{~s}$ e $3,59 \mathrm{~s}$.

\subsection{3 - Sintonia do controlador PID adaptativo segundo método de ASTRÖM \& HÄGGLUND [1]}

Através da oscilação sustentada obtida de cada ensaio, referentes às vazões de 300, 400,500, $600 \mathrm{e}$ $700 \mathrm{~L} / \mathrm{h}$ foram obtidos os valores da intensidade desta oscilação (2I) para cada vazão. Para ilustrar a oscilação sustentada obtida nos ensaios, a Figura 6 mostra o comportamento da variação de temperatura $\left({ }^{\circ} \mathrm{C}\right)$ e da potência da resistência da linha (\%) para a vazão de 300L/h.

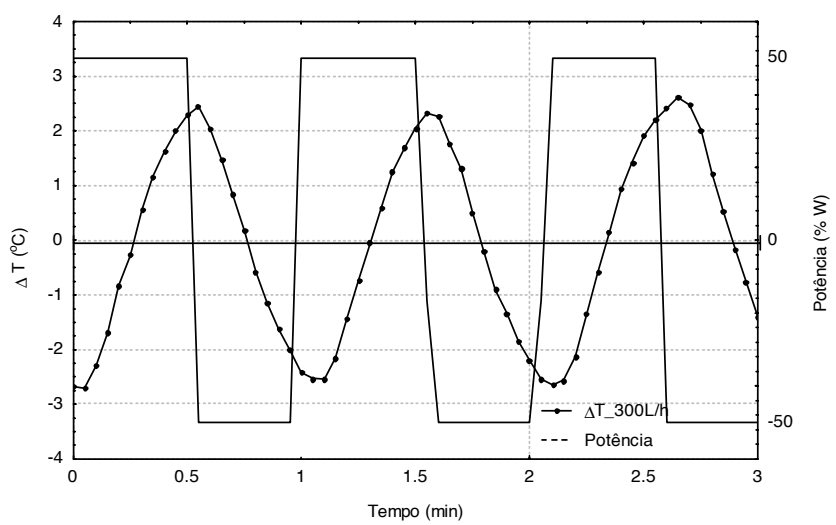

FIGURA 6. Oscilação sustentada obtida na vazão de 300L/h

TABELA 6. Constantes do controlador PID pelo o método de ASTRÖM \& HÄGGLUND [1]

\begin{tabular}{lccccc}
\hline \multirow{2}{*}{ Parâmetro } & \multicolumn{5}{c}{ Vazão do fluido (L/h) } \\
\cline { 2 - 6 } & 300 & 400 & 500 & 600 & 700 \\
\hline $2 \mathrm{I}\left({ }^{\circ} \mathrm{C}\right)$ & 4,92 & 3,92 & 3,26 & 3,17 & 2,73 \\
$\mathrm{Pcr}(\mathrm{s})$ & 0,95 & 0,95 & 0,97 & 1,05 & 1,00 \\
$2 \mathrm{~h}(\% \mathrm{~W})$ & 100 & 100 & 100 & 100 & 100 \\
$\mathrm{Kcr}\left({ }^{\circ} \mathrm{C} / \% \mathrm{~W}\right)$ & 25,88 & 32,48 & 39,06 & 40,17 & 46,64 \\
$\mathrm{Kc}\left({ }^{\circ} \mathrm{C} / \% \mathrm{~W}\right)$ & 15,53 & 19,49 & 23,43 & 24,10 & 27,98 \\
$\tau_{\mathrm{i}}(\mathrm{s})$ & 28,53 & 28,5 & 29,10 & 31,50 & 30,00 \\
$\tau_{\mathrm{d}}(\mathrm{s})$ & 7,13 & 7,13 & 7,28 & 7,88 & 7,50 \\
\hline
\end{tabular}

Os valores da intensidade da perturbação e da constante proporcional do controlador PID, calculadas conforme a Tabela 2 são mostradas na Tabela 6. Devido à freqüência de imposição da perturbação ter sido de 30s, os valores do período crítico para cada vazão foram similares, e portanto geraram valores das constantes $t_{i}$ e $\tau_{\mathrm{d}}$ próximos. Devido à proximidade destes valores, os parâmetros $\tau_{\mathrm{i}}$ e $\tau_{\mathrm{d}}$ foram considerados constantes, nos valores de $30 \mathrm{~s}$ e $7,5 \mathrm{~s}$.

A Figura 7 mostra o ajuste dos valores do parâmetro ganho do controlador $\left(\mathrm{K}_{\mathrm{c}}\right)$ à equação de primeiro grau em função da vazão do fluido. 


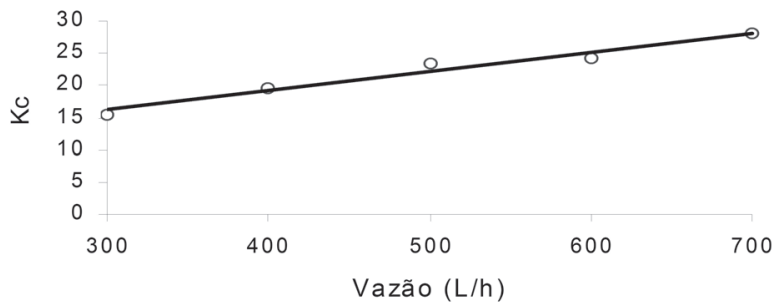

FIGURA 7. Ajuste da função do valor de $K_{c}$ em função da vazão de fluido (Método "Bang-Bang")

A função deste ajuste é mostrada na equação (5).

$\mathrm{K}_{\mathrm{c}}=2,95 \cdot 10^{-2} \mathrm{~V}_{\mathrm{aq}}+7,35 \quad \mathrm{R}^{2}=0,9640$

\section{3 - Comparação de desempenho dos controladores}

Depois de implementadas as constantes dos controladores PID com os valores médios e as funções adaptativas da curva de reação e de ASTRÖM \& HÄGGLUND [1] foram realizadas as perturbações na vazão, descritas na Tabela 3, para constatação da eficácia dos mesmos.

As Tabelas 7, 8 e 9 mostram os valores dos índices de erros (IAE, ISE e ITAE) calculados para cada sintonia, o tempo e erro de sobre-sinal $\left(\mathrm{t}_{\mathrm{s}} \mathrm{e}_{\mathrm{s}}\right)$ e o erro no tempo de 2,7 minutos ( $\left.\mathrm{e}_{\infty}\right)$.

TABELA 7. Índices de erros do controlador adaptativo conforme a curva de reação do processo

\begin{tabular}{lcccccc} 
& $\begin{array}{c}\text { IAE } \\
\left({ }^{\circ} \mathrm{C}\right)\end{array}$ & $\begin{array}{c}\text { ISE } \\
\left({ }^{\circ} \mathrm{C}^{2}\right)\end{array}$ & $\begin{array}{c}\text { ITAE } \\
\left({ }^{\circ} \mathrm{C} . \mathrm{min}\right)\end{array}$ & $\begin{array}{c}\mathrm{t}_{\mathrm{s}} \\
(\mathrm{min})\end{array}$ & $\begin{array}{c}\mathrm{e}_{\mathrm{s}} \\
\left({ }^{\circ} \mathrm{C}\right)\end{array}$ & $\begin{array}{c}\mathrm{e}_{\mathrm{o}} \\
\left({ }^{\circ} \mathrm{C}\right)\end{array}$ \\
\hline Perturbação de 500-400 L/h & 0,60 & $8,51.10^{5}$ & 0,87 & 0,32 & 0,30 & 0,20 \\
Perturbação de 500-450 L/h & 0,40 & $3,94.10^{3}$ & 0,65 & 0,10 & 0,10 & $-0,30$ \\
Perturbação de 500-550 L/h & 0,50 & $6,68.10^{3}$ & 0,72 & 1,12 & $-0,50$ & $-0,40$ \\
Perturbação de 500-600 L/h & 0,57 & $7,45.10^{3}$ & 0,78 & 1,68 & $-0,40$ & $-0,10$ \\
Médias & 0,52 & $6,67.10^{3}$ & 0,76 & & &
\end{tabular}

TABELA 8. Índices de erros do controlador com as constantes nos valores médios da curva de reação do processo

\begin{tabular}{lcccccc}
\hline & $\begin{array}{c}\text { IAE } \\
\left({ }^{\circ} \mathrm{C}\right)\end{array}$ & $\begin{array}{c}\text { ISE } \\
\left({ }^{\circ} \mathrm{C}^{2}\right)\end{array}$ & $\begin{array}{c}\text { ITAE } \\
\left({ }^{\circ} \mathrm{C} . \mathrm{min}\right)\end{array}$ & $\begin{array}{c}\mathrm{t}_{\mathrm{s}} \\
(\mathrm{min})\end{array}$ & $\begin{array}{c}\mathrm{e}_{\mathrm{s}} \\
\left({ }^{\circ} \mathrm{C}\right)\end{array}$ & $\begin{array}{c}\mathrm{e}_{\infty} \\
\left({ }^{\circ} \mathrm{C}\right)\end{array}$ \\
\hline Perturbação de $500-400 \mathrm{~L} / \mathrm{h}$ & 1,12 & $2,36.10^{-2}$ & 1,97 & 2,67 & 1,10 & 0,80 \\
Perturbação de $500-450 \mathrm{~L} / \mathrm{h}$ & 0,52 & $4,77.10^{-3}$ & 0,72 & 0,20 & 0,30 & 0,00 \\
Perturbação de 500-550 L/h & 0,40 & $3,23.10^{-3}$ & 0,59 & 0,78 & $-0,30$ & 0,20 \\
Perturbação de 500-600 L/h & 0,47 & $5,00.10^{-3}$ & 0,68 & 0.45 & $-0,40$ & $-0,10$ \\
Médias & 0,63 & $9,15.10^{-3}$ & 0,99 & & &
\end{tabular}

TABELA 9. Índices de erros do controlador adaptativo conforme ASTRÖM \& HÄGGLUND [1]

\begin{tabular}{lcccccc}
\hline & $\begin{array}{c}\mathrm{IAE} \\
\left({ }^{\circ} \mathrm{C}\right)\end{array}$ & $\begin{array}{c}\mathrm{ISE} \\
\left({ }^{\circ} \mathrm{C}^{2}\right)\end{array}$ & $\begin{array}{c}\mathrm{ITAE} \\
\left({ }^{\circ} \mathrm{C} \cdot \mathrm{m} \text { in }\right)\end{array}$ & $\begin{array}{c}\mathrm{t}_{\mathrm{s}} \\
(\mathrm{min})\end{array}$ & $\begin{array}{c}\mathrm{e}_{\mathrm{s}} \\
\left({ }^{\circ} \mathrm{C}\right)\end{array}$ & $\begin{array}{c}\mathrm{e}_{\infty} \\
\left({ }^{\circ} \mathrm{C}\right)\end{array}$ \\
\hline Perturbação de $500-400 \mathrm{~L} / \mathrm{h}$ & 0,41 & $6,12 \cdot 10^{-3}$ & 0,43 & 0,33 & 0,40 & $-0,20$ \\
Perturbação de $500-450 \mathrm{~L} / \mathrm{h}$ & 0,28 & $2,13 \cdot 10^{-3}$ & 0,33 & 0,53 & 0,20 & $-0,10$ \\
Perturbação de $500-550 \mathrm{~L} / \mathrm{h}$ & 0,42 & $6,67 \cdot 10^{-3}$ & 0,66 & 1,57 & $-0,40$ & $-0,20$ \\
Perturbação de 500-600 L/h & 0,84 & $1,86 \cdot 10^{-2}$ & 1,21 & 1,78 & $-0,40$ & $-0,30$ \\
Médias & 0,49 & $8,38 \cdot 10^{-3}$ & 0,66 & & & \\
\hline
\end{tabular}

As Figuras 8 a 13 mostram, para os três controladores configurados, o comportamento da variação de temperatura após perturbações no sistema de intensidades, conforme a Tabela 3 , isto é, de $500 \mathrm{~L} / \mathrm{h}$ para 450 , 550,500 e $600 \mathrm{~L} / \mathrm{h}$, juntamente com o comportamento da potência da resistência.

Analisando as tabelas dos índices de erros de cada controlador e as figuras que ilustram o desempenho dos mesmos, verifica-se que o controle adaptativo realizado com a sintonia de ASTRÖM \& HÄGGLUND [1] apresentou menores valores tanto do erro de sobre-sinal, $\mathrm{e}_{\mathrm{s}}$ (overshoot), como dos 3 índices de erro. Além disso, neste controle, o comportamento da variável manipulada (potência da resistência) apresentou menores oscilações no controle da temperatura do fluido. Mesmo assim, as outras duas sintonias não podem ser consideradas totalmente insatisfatórias, pois a oscilação da temperatura (DðT) em torno do set-point ficou dentro da faixa de $\pm 1^{\circ} \mathrm{C}$ na maioria dos ensaios realizados.

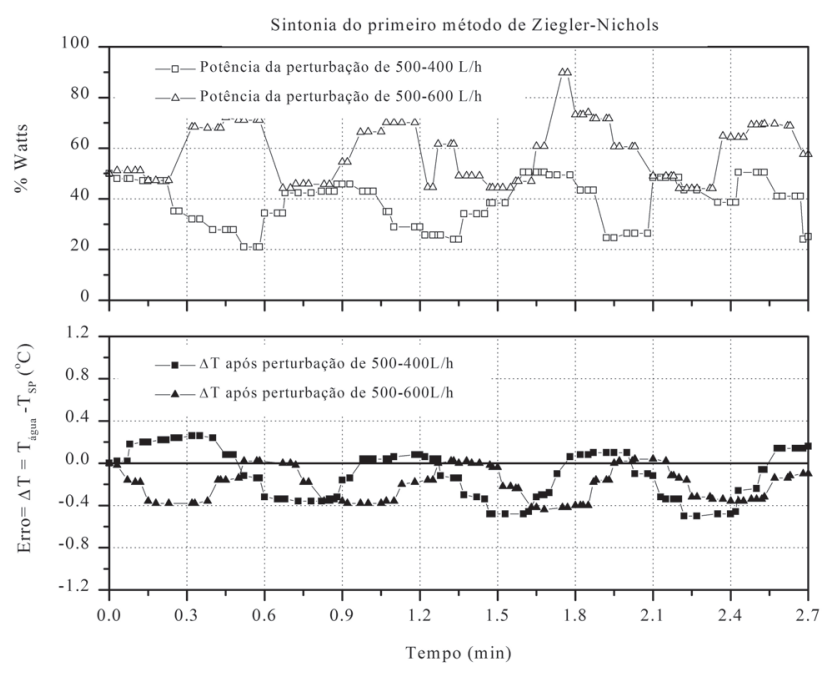

FIGURA 8. Desempenho do controlador adaptativo conforme sintonia da curva de reação após perturbações de $500 \mathrm{~L} / \mathrm{h}$ para 550 e $450 \mathrm{~L} / \mathrm{h}$.

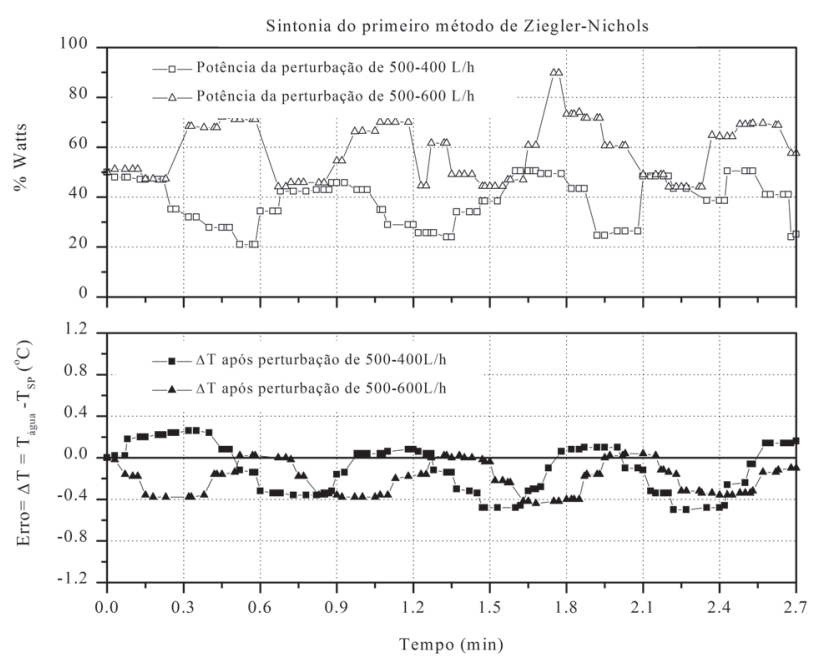

FIGURA 9. Desempenho do controlador adaptativo conforme sintonia da curva de reação após perturbações de $500 \mathrm{~L} / \mathrm{h}$ para 400 e $600 \mathrm{~L} / \mathrm{h}$ 


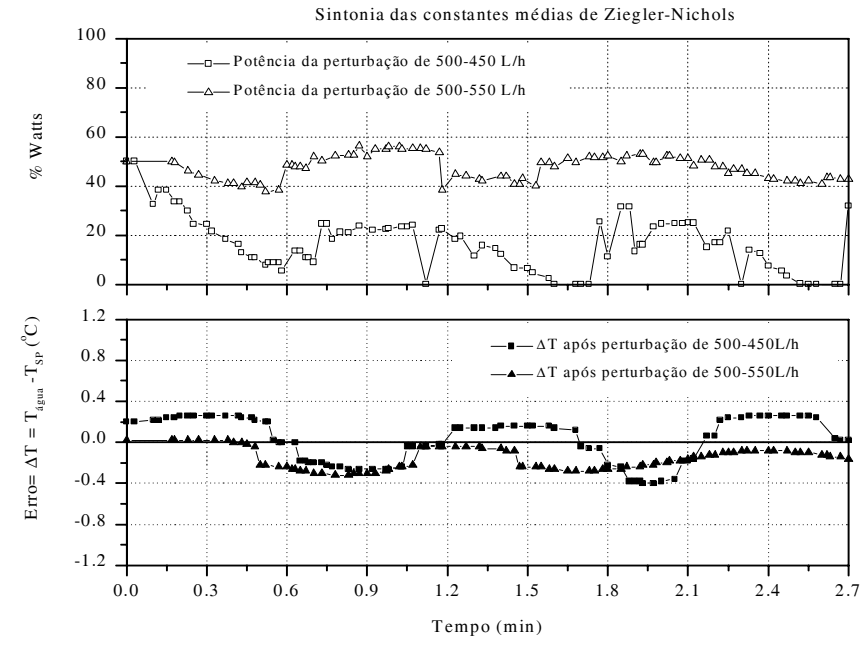

FIGURA 10. Desempenho do controlador adaptativo com as constantes nos valores médios após perturbação de 500L/h para 550 e $450 \mathrm{~L} / \mathrm{h}$
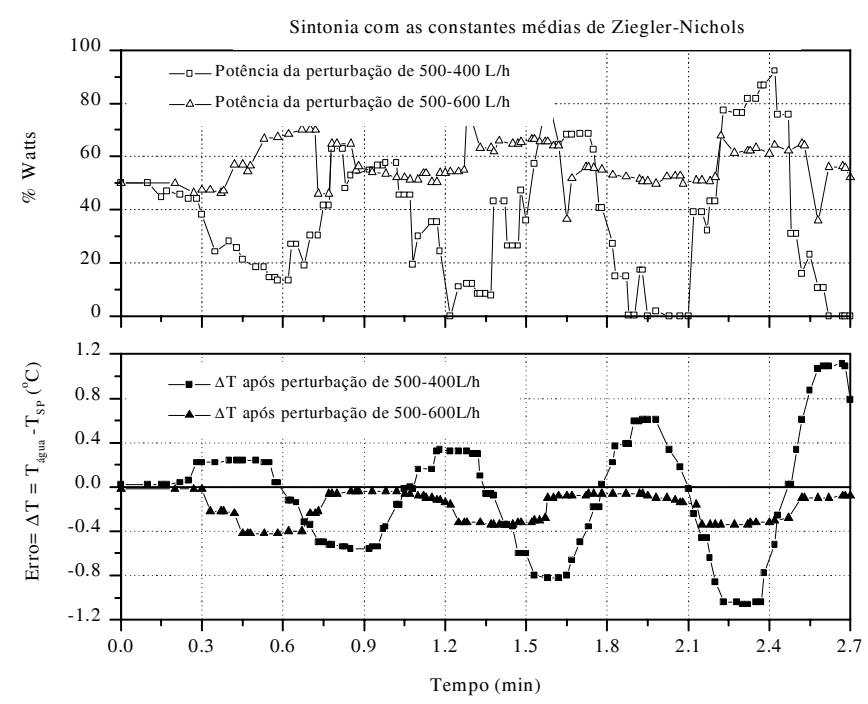

FIGURA 11. Desempenho do controlador adaptativo com as constantes nos valores médios após perturbação de $500 \mathrm{~L} / \mathrm{h}$ para 600 e $400 \mathrm{~L} / \mathrm{h}$
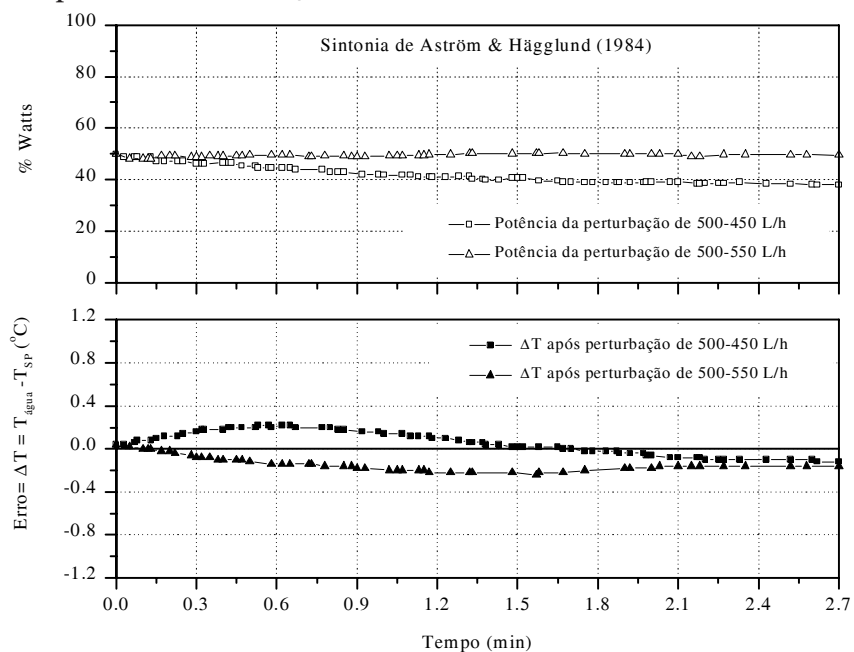

FIGURA 12. Desempenho do controlador adaptativo de ASTRÖM \& HÄGGLUND [1] após perturbação de 500L/h para 450 e $550 \mathrm{~L} / \mathrm{h}$

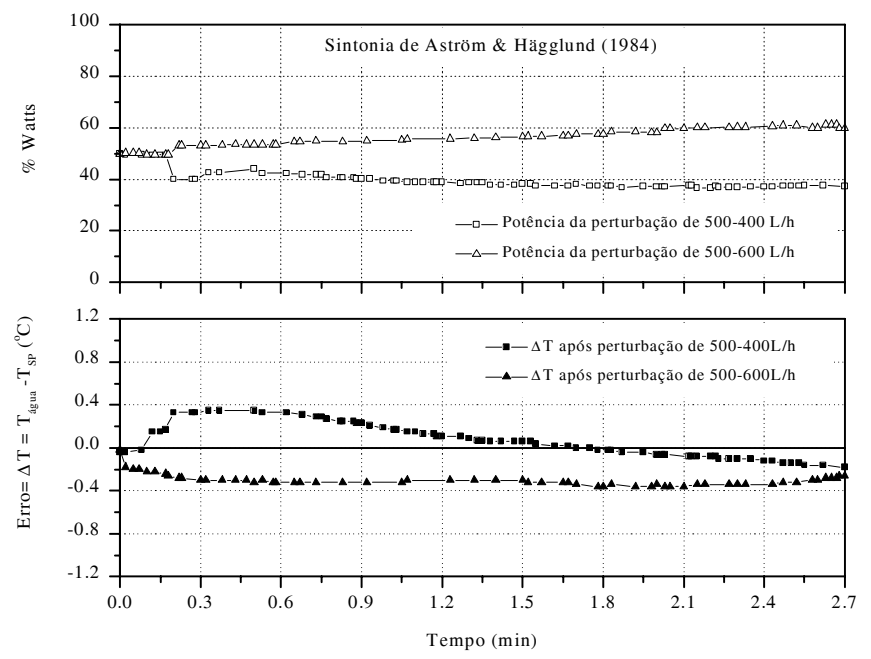

FIGURA 13. Desempenho do controlador adaptativo de ASTRÖM \& HÄGGLUND [1] após perturbação de 500L/h para 400 e $600 \mathrm{~L} / \mathrm{h}$

\section{4 - CONCLUSÕES}

Este trabalho consistiu em avaliar o desempenho de três sintonias para o controlador PID para o controle da água de aquecimento de um processo de pasteurização: adaptativo da curva de reação, parâmetros com valores médios, e adaptativo de ASTRÖM \& HÄGGLUND [1]. Os resultados mostraram que todas sintonias testadas apresentaram valores de erros dentro da faixa de $\pm 1^{\circ} \mathrm{C}$. Porém, através da comparação dos índices de erros (IAE, ISE, ITAE) e dos valores dos erros de sobressinal constatou-se que a sintonia pelo método adaptativo de ASTRÖM \& HÄGGLUND [1] apresentou menores valores de índices de erros e de erro de sobressinal. Como não foi realizado nenhum ajuste dos parâmetros calculados com cada sintonia, conclui-se que o método de sintonia de ASTRÖM \& HÄGGLUND [1], dentre os testados, é o que proporciona um controle de menores oscilações e que, no caso de optar-se por um ajuste fino dos parâmetros do controlador, seria o método que necessitaria de menores alterações.

\section{5 - REFERÊNCIAS BIBLIOGRÁFICAS}

[1] ASTRÖM, K.J.; HÄGGLUND, T. Automatic tuning of simple regulators with specifications on phase and amplitude margins. Automatica, v. 20, n. 5, p. 645$651,1984$.

[2] BERTO, M.I. Avaliação experimental do controle da pasteurização contínua de um fluido modelo de suco de laranja. Campinas, 2004. 271p. Tese (Doutor em Engenharia de Alimentos). Departamento de Engenharia de Alimentos, Universidade Estadual de Campinas (UNICAMP).

[3] CORREA NETO, R.D.S. Processamento de suco de laranja pasteurizado em garrafas depolietileno tereftalato (PET). Campinas, 1998. 88p. Dissertação (Mestre em Tecnologia de Alimentos). Tecnologia de Alimentos, Universidade Estadual de Campinas, (UNICAMP). 
[4] COUGHANOWR, D.R.; KOPPEL, L.B. Análise e controle de processos. Tradução de Carlos Augusto G. Perlingeiro e outros. Guanabara, Rio de Janeiro. 1978, 474p.

[5] EARGMAN, B.A.; ROUSE, A.H. Heat inactivation temperature-time relationships for pectinesterase inactivation in citrus juice. Journal of Food Science, v. 41, n. 6, p. 1396-1397, 1976.

[6] IBARROLA, J.J.; GUILLÉN, J.C.; SANDOVAL, J.M.; GARCÍA-SANZ, M. Modelling of a High temperature short time pasteurization Process. Food Control, v. 9, n. 5, p. 267-277, 1998.

[7] KIMBALL, D.A. Citrus processing: quality control and technology. Van nostrand Reinhold, New York. 1991, $473 p$.

[8] NEGIZ, A.; CINAR, A.; SCHLESSER, J.E.; RAMANAUKAS, P.; ARMSTRONG, D.J.; STROUP, W. Automated Control of high temperature short time pasteurization. Food Control, v. 7, n. 6, p. 309-315, 1996.

[9] OGUNNAIKe, B.A.; RAY, W.H. Process dynamics, modeling control. Oxford University Press, New York, Oxford. 1994, 1260p.

[10] SCHLESSER, J.E.; ARMSTRONG, D.J.; CINAR, A.; RAMANAUKAS, P.; NEGIZ, A. Automated Control and Monitoring of Thermal processing using High Temperature short time Pasteurization. Journal of Dairy Science, v. 80, n. 10, p. 2291-2296, 1997.

[11] SILVA, F.V.D. Comparação do desempenho de um sistema de refrigeração para resfriamento de líquido controlado a diferentes modos de controle. Campinas, 2003. 327p. Tese (Doutor em Engenharia de Alimentos). Departamento de Engenharia de Alimentos, Universidade Estadual de Campinas (UNICAMP).

\section{6 - AGRADECIMENTOS}

Os autores agradecem à Fundação de Amparo à Pesquisa do Estado de São Paulo - FAPESP (00/00437-0) pelo apoio financeiro. 